\title{
AUTOCONTROL Y COMPORTAMIENTO: EFECTIVIDAD DE LAS TECNICAS AUTOINSTRUCCIONALES EN EL APRENDIZAJE DE LAS OPERACIONES ARITMETICAS
}

COMAS i MONGAY, M.D.

Escola Universitària de Mestres de Vic

\section{SUMMARY}

This experiment was designed to research the efficacy of self-instructional variables to improve performance in solving easy arithmetic problems. Children, boys and girls, six and seven years old, studying first school year of E.G.B., were used.

The results, as a whole, tell us that training in self-instructional techniques significantly affects the dependent variable, decreasing the number of mistakes pupils make in arithmetical operations. It is appropriate to underline the group verbalizing the addition, which got the most significant reduction. These results, which confirm the findings of other authors, show the possibility of applying Cognitive-conductual techniques in the school frame.

\section{INTRODUCCION}

Dentro del Análisis del Comportamiento, que es el marco reterencial de nuestro estudio, se experimentó a finales de los años 60 y principios de los 70 un período de cambio que apuntó hacia nuevas líneas de investigación, proponiendo nuevas hipótesis y manipulando varibles no exploradas hasta entonces. (Bayés, 1980; Genovard, Gotzens y Montané, 1981; Mahoney, 1981; Meichenbaum, 1981).

Esta evolución se caracteriza, desde nuestro punto de vista, por el intento de encontrar las variables relevantes que hagan posible predecir el control que uno mismo tiene sobre su propio comportamiento, es decir el autocontrol. Estas variables incluyen comportamientos abiertos, como son la autoobservación, autoevaluación, autoreforzamiento, etc. Y comportamientos encubiertos, los cuales permiten analizar y controlar experimentalmente la modalidad cognitiva y respuesta, como son las espectativas, la imaginación, los pensamientos, las autoinstrucciones, los reforzamientos encubiertos, etc... Muchos han sido los autores que en la década de los sesenta contribuyeron, con estos planteamientos a favorecer el comportamiento de autocontrol de nifios y adultos (Bandura y Kupers, 1963; Bandura y Perloff, 1967, Bandura, 1969; Bijou y Baer, 1961; Cautela, 1966,1970; Homme, 1965; Kanfer, 1966; Kanfer y Phillips, 1970; Mahoney, 1970; Meichenbaum y Goodman, 1971; Rachlin, 1970).

Destacamos aqui, de estas nuevas líneas de investigación, el trabajo de Meichenbaum y Goodman, (1971) el cual marca el camino a seguir para analizar experi- mentalmente lo que se ha llamado estrategias cognitivoconductuales: Autoinstrucciones, que es el objeto de estudio del trabajo que presentamos.

De acuerdo con Meichenbaum (1981) entendemos por autoinstrucciones imágenes y afirmaciones verbales hacia uno mismo, las cuales apoyan, dirigen o mantienen el comportamiento.

Los antecedentes de estas ideas los podemos encontrar en la obra de Jean Piaget y Lev S. Vigotsky (Luria, 1975; Pinillos, 1975). Más recientemente en un discfpulo de Vigotsky, A.R. Luria (1959-1961) el cual demuestra que los niños pequeños hacen uso de un lenguaje interno «egocéntrico» para controlar su comportamiento.

Estudios más recientes han demostrado la eficacia del "paquete» autoinstruccional en el ámbito escolar (tanto en escuelas "especiales» como "ordinarias") en relación a distintos problemas, aumentando el tiempo de atencion y dedicación a las tareas de niños etiquetados de hiperactivos, impulsivos, agresivos (Bomstein y Quevillon, 1976; Kendall y Wilcox, 1980; Meichenbaum y Gooddam, 1971); facilitando el autocontrol de «resistencia a la tentación» (Mischel y Patterson, 1976; Newman y Kanfer, 1976). En el ámbito familiar, mejorando las relaciones familiares padres e hijos; disminuyendo comportamientos perturbadores (Denicola y Sandler, 1981; Sanders y Glynn, 1981).

En el ámbito escolar, de los estudios que evalúan la precisión en los aprendizajes académicos, nos fijaremos 
más detalladamente en los que han intentado encontrar relaciones funcionales entre la autoverbalización de instrucciones y la actuación en matemáticas.

Según ello, Lovitt y Curtis (1986) en un estudio claro y sencillo, propusieron la manipulación experimental que consistió en leer un problema matemático en voz. alta, «algún número menos dos, igual a seis» $(-2=$ 6), y después escribir la respuesta. Encontraron efectividad en reducir la tasa de errores de las operaciones. O sea, lo que proponen para mejorar la actuación aritmética es la autoverbalización del problema antes de resolverlo. Burgio, Whitman y Johnson, (1980) entrenando a verbalizar un paquete autoinstruccional, durante cada operación, observaron una disminución en el número de errores en sumas y restas de uno y dos digitos. Spates y Kanfer (1977) usan la autoverbalización de instrucciones intentando averiguar qué com- ponentes del procedimiento autoinstruccional afectan significativamente a la variable dependiente, número de errores en las operaciones de sumar. Los autores informan haber encontrado la máxima reducción del número de errores en las sumas, en el grupo llamado de "establecimiento de criterio», seguido con poca diferencia de los grupos de "autoevaluacion», y «autoevaluación más autoreforzamiento", no encontrando diferencias significativas en el grupo de «autoobservación».

Nuestro estudio parte de la propuesta de manipulación experimental formulada por Spates y Kanfer (1977) pero, y de acuerdo con O'Leary y Dubey (1979), no para evaluar el modelo de autoregulación (Kanfer 1970, 1975) sino para intentar determinar la relación funcional de las variables autoinstruccionales. Para hacerlo, tuvimos que realizar juntamente con el experimento inicial un experimento complementario para poder así resolver unos errores metodológicos que surgieron en el entrenamiento de los procedimientos autoinstraccionales.

Nuestros propósitos en la investigación que presentamos fueron los siguientes:

a) Evaluar la eficacia de los procedimientos autoinstruccionales en el mejoramiento de la resolución de operaciones aritméticas sencillas.

b) Observar si la reducción del número de errores en las sumas de los estudiantes que hayan recibido entrenamiento en autoverbalizacion de instrucciones, es significativamente mayor que la de los que no hayan recibido entrenamiento.

c) Ver cómo cada una de las diferentes autoinstrucciones que presentamos, afecta la variable dependiente.

En resumen, la hipótesis que proponemos es que el entrenamiento con las tecnicas de autoverbalización de instrucciones mejora el rendimiento en los aprendizajes de operaciones aritméticas sencillas.

Dentro de las técnicas de autocontrol, consideramos que el aprendizaje de las técnicas autoinstruccionales puede ser uno de los caminos para demostrar la posjble aplicación de la orientación cognitivo-conductual en el campo educativo.

\section{METODO}

\section{Sujetos}

Los sujetos $\left(\mathrm{S}_{\mathrm{s}}\right) \operatorname{seran} 220$ niños y niñas, de una edad comprendida entre los seis y siete años, que estaban realizando durante el año académico 80/81 el primer curso de E.G.B. en cinco escuelas de la comarca de Osona

Del grupo original de 220 nif̂tos, participaron en el experimento, mejor dicho, pasaron a la fase de entrenamiento, los 96 que alcanzaron las premisas establecidas en la Fase 1 (Pre-test). De éstos hubo siete que por diversas causas escolares no pudieron participar íntegramente en el experimento y no se les tuvo en cuenta. Asi pues, quedaron como sujetos experimentales $89 \mathrm{ni}$ ños y niñas.

En el experimento complementario participaron 20 niños. Después del pre-test se escogieron 14 para pasar a la fase de entrenamiento.

Los experimentadores (Es.) fueron 11 estudiantes de Tercer curso de la Escuela Universitaria Balmes, de formación del profesorado de E.G.B. (Vic), coordinados por el profesor de psicología de este mismo curso.

\section{Diseño experimental}

Para el experimento utilizamos un diseno intergrupo: Pre-test-Postest-Control, con cinco grupos. Los procedimientos de autocontrol que se evaluaron son cuatro variantes autoinstruccionales que constituyen, junto con el grupo control, los cinco grupos experimentales.

Verbalización de la suma (A)

Verbalización del orden a seguir para efectuar la operación de sumar (B)

$A+B$ : Verbalizacion de la suma y del orden que se ha de seguir para efectuarla (C)

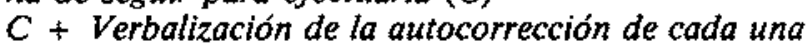
de las sumas (D)

Control (E).

Los nitos fueron distribuidos al azar en cada uno de Ios grupos experimentales; se segula una lista que comenzaba por los sujetos que hablan realizado menos errores en el Pre-test y acababa por los que habfan hecho un número más elevado de ellos. De esta manera, los niños y niñas quedaban repartidos más equitativamente. En cada escuela había niffos de los cinco grupos experimentales. Los experimentadores se repartieron por las cuatro escuelas siguiendo como único criterio preestablecido que el E. no conociese a los SS. y entrenaba indistintamente tanto a Ss. de un grupo como a Ss. de los otros cuatro grupos experimentales. 


\section{Procedimiento}

El procedimiento es muy similar al del estudio ya comentado de Spates y Kanfer (1977). L.as variantes que hemos introducido han sido motivadas por la traducción y adaptación al catalán de los textos trabajados, y también, por problemas prácticos en la aplicación a nuestro contexto educativo.

A los Ss. se les veia individualmente en una habitación próxima a la clase en que se trabajaban las tres fases del experimento: 1. Pre-test; 2. Entrenamiento; 3. Postest.

Entre las fases 1 y 2 pasaba un dia escolar y, durante ese dia, los Sujetos no recibian ningún tipo de entretenimiento en sumas. La fase 3 se realizaba inmediatamente después de la fase 2.

Para homologar la interacción de los diferentes Es. con los chicos, cada uno de ellos tenía unas hojas con unas pautas que explicaban minuciosamente lo que tenian que decir y hacer en cada momento y en cada caso.

Para tener una medida de fiabilidad de la intervención de los Es., las fases de Entrenamiento de cada sesión se registraban en cintas magnetofónicas.

Para confeccionar las pautas a seguir en el procedimiento, partiamos de una experiencia piloto de toda la mecánica del experimento que nos permitió matizar, rectificar, anadir o eliminar aspectos del planteamiento inicial. Las que presentábamos en este estudio son las definitivas.

\section{Fases del experimento}

Fase 1: Pre-test

A cada niño se le pedía, siguiendo unas instrucciones determinadas, que completase una hoja de sumas, la dificultad de las cuales, iba de acuerdo con el nivel que proponia la/el maestro/a, a quien se consultaba previamente.

Durante esta fase el E. hacia ver que estaba repasando otra cosa. Para hacer esta hoja el niño/a disponia de un tiempo ilimitado y cuando interrumpfa su trabajo por algún motivo, se le decfa: "ves haciéndolo, tal como sepas".

Si hacía las 12 sumas bien, se le ponian 12 sumas más de un grado más difícil, siguiendo la gradación de sumas ordenadas en dificultad creciente, propuestas por Backhol (1981).

Si las hacia mal, se le daba otra hoja, pero, esta vez, de un grado de dificultad inferior, siguiendo la misma gradación.

Ast, intentamos encontrar el grado de dificultad en que los niffos hiciesen de dos a diez errores, los dos inclusives, con la finalidad de obtener, para la fase 2 , una muestra de niffos susceptibles a las intervenciones conductuales (chicos que saben hacer sumas pero que pue- den mejorar - que hacen de cinco a diez bien-, y chicos que saben minimamente, que hacen de dos a cuatro bien).

Hubo niños que no se situaron en estos criterios, por diversas causas. Estos quedaban eliminados y no pasaban a la tase siguiente.

\section{Fase 2: Entrenamiento}

En unas hojas en las que habia ocho sumas, los Es., siguiendo las instrucciones pertinentes, enseñaban las autoverbalizaciones correspondientes a cada uno de los Ss. de los diversos grupos experimentales.

Para amoldar las autoverbalizaciones seguíamos los siguientes pasos:

1. El E. hacía la demostración de lo que se tenía que decir mientras efectuaba la suma; a continuación el Ss. tenia que repetir, punto por punto, lo que habla dicho el experimentador, efectuando la primera suma de la hoja de entrenamiento.

2. Verbalizaban la operación en voz alta los dos juntos, E. y S.

3. EI S. lo hacfa solo y el E. le corregía oportunamente. Durante todo el proceso se amoldaban las verbalizaciones, no los resultados de la operación aritmética.

De acuerdo con el nivel que determinábamos en el pretest a los niños se les entrenaba en unas u otras verbalizaciones según el tipo de suma.

Consideramos a los niños suficientemente entrenados cuando verbalizaron correctamente las instrucciones en tres sumas consecutivas, independientemente de si el resultado de la suma era correcto o no.

\section{Fase 3: Postest}

Inmediatamente después de la fase de entrenamiento, repartiamos a todos los Sujetos una hoja con 12 sumas, tal como se habia hecho cuando se entrenaban, y se les decia: «Ya puedes empezar; cuando acabes, dimelon. Finalizaba el postest cuando entregaba la hoja con todas las sumas resueltas.

Para reafirmar los resultados obtenidos se evaluaron complementariamente sólo los grupos experimentales C y D que corresponden a "Verbalización de la suma y del orden que se ha de seguir para hacerla»; "C verbalización de la autocorrección de cada una de las sumas». Seguimos, con todo detalle, el mismo planteamiento.

\section{Medidas dependientes}

La variable dependiente que hemos medido en nuestro experimento es el número de errores cometides en una hoja de 12 sumas.

Considerábamos error cada una de las sumas mal hechas. O sea, la variable dependiente era el conjunto de 
las variaciones observadas en el número de errores del Postest de cada uno de los Ss. de la muestra experimental. La diferencia de estas puntuaciones con las obtenidas en el pre-test nos permitirá ver si el entrenamiento ha actuado para favorecer el aprendizaje disminuyendo, en consecuencia, el número de errores.

\section{RESULTADOS}

Los resultados obtenidos, por la variable dependiente según la media de errores antes y después del"entrenamiento, los podemos observar en la Figura 1. La lectura de éstos nos dice que el grupo que consigue la reducción más alta en número de errores es el grupo (A), con una diferencia entre medias de 2.74. En segundo lugar se encuentra el grupo (C) con una diferencia de 2.47; el tercero es el grupo (B) con una diferencia de 1.12; el cuarto el grupo control con 1.11 de diferencia y, en último lugar, se encuentra el grupo (D), con una diferencia de 0.62 . Teniendo en cuenta los valores del experimento complementario, el grupo (D) con una reducción de la media de errores de 2.23 , se sitúa en segundo lugar, al lado del grupo (C), que se mantiene igualmente en esta posición.

Estadísticamente, aplicando un análisis de covarianza que evalúa los efectos del tratamiento sobre el número de errores finales, teniendo en cuenta el número de errores iniciales para cada individuo, obtenemos relaciones estadísticamente significativas por el factor tratamiento $F(4,84)=2.46 ; p<.05$. De este modo, podemos decir que el tratamiento afecta significativamente a los resultados (número de errores finales).

En la Tabla 1 comparamos, mediante la prueba $t$ de Tukey, los resultados obtenidos por los grupos que han recibido entrenamiento con el grupo control de notratamiento. Este análisis indica que el único grupo que consiguió una mejora significativa fue el grupo (A), p $<.05$ y que hay una tendencia a la significacion en el grupo (C) y (D) -experimento complementario--, $(\mathrm{p}<.10$ y $\mathrm{p}<.10)$. En el grupo (B), no observamos diferencias significativas $(p<90)$ respecto el grupo control.

\section{DISCUSION}

Como hemos podido observar en los resultados, la variable tratamiento, o sea, el entrenamiento en las técnicas autoinstruccionales, en conjunto, afecta significativamente la variable dependiente, disminuyendo el número de errores que hacen los alumnos en las operaciones aritméticas, confirmando así los resultados de Spates y Kanfer (1977).

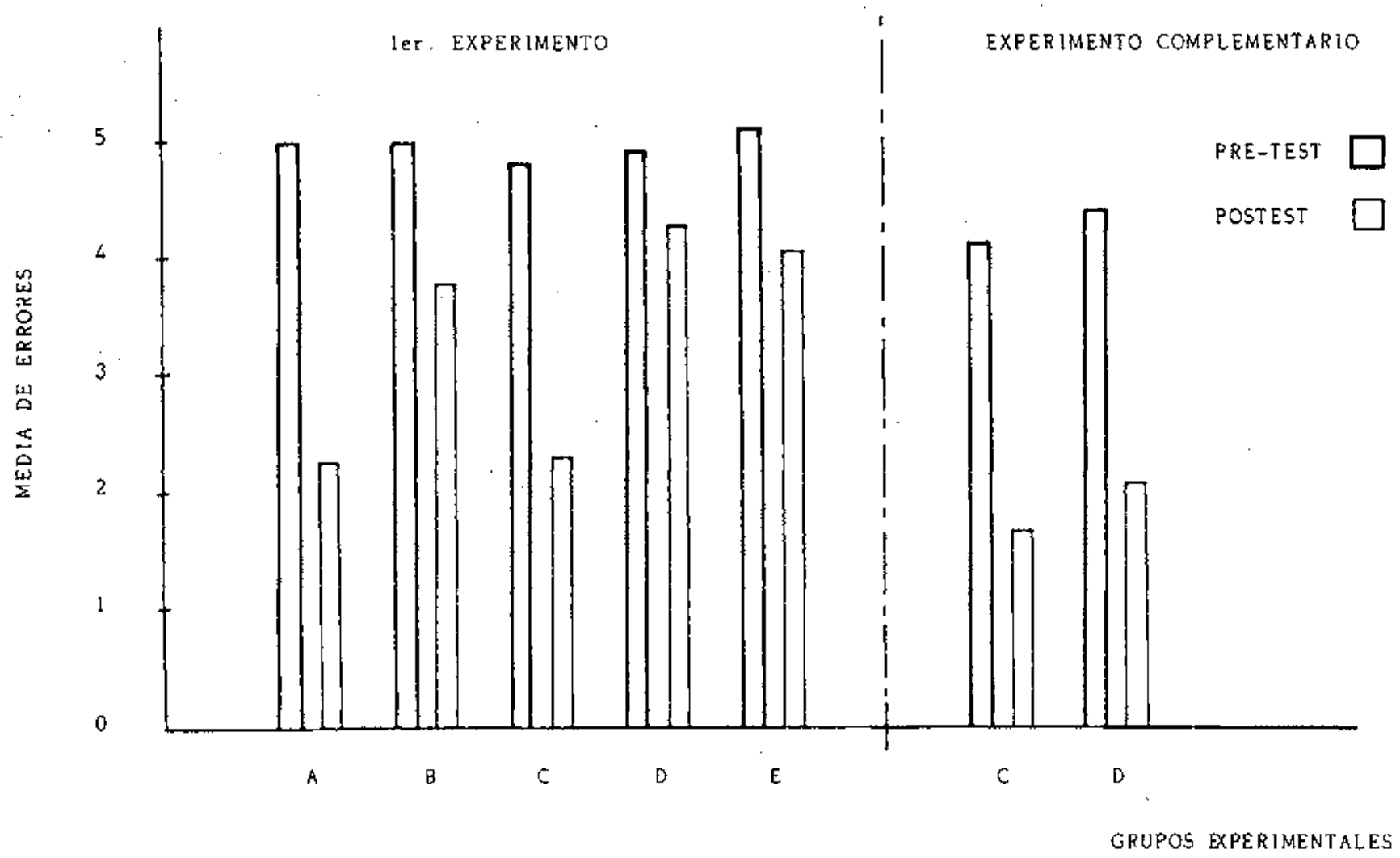

fig. 1 Media de error Pretest - Postest en función del tipo de entrenamiento recibido por los diferentes grupos experimentales. 
Tabla !

Comparación del número de errores final entre los grupos entrenados y el control, y jos grupos entrenados entre etlos, con la prueba $t$ de Tukey.

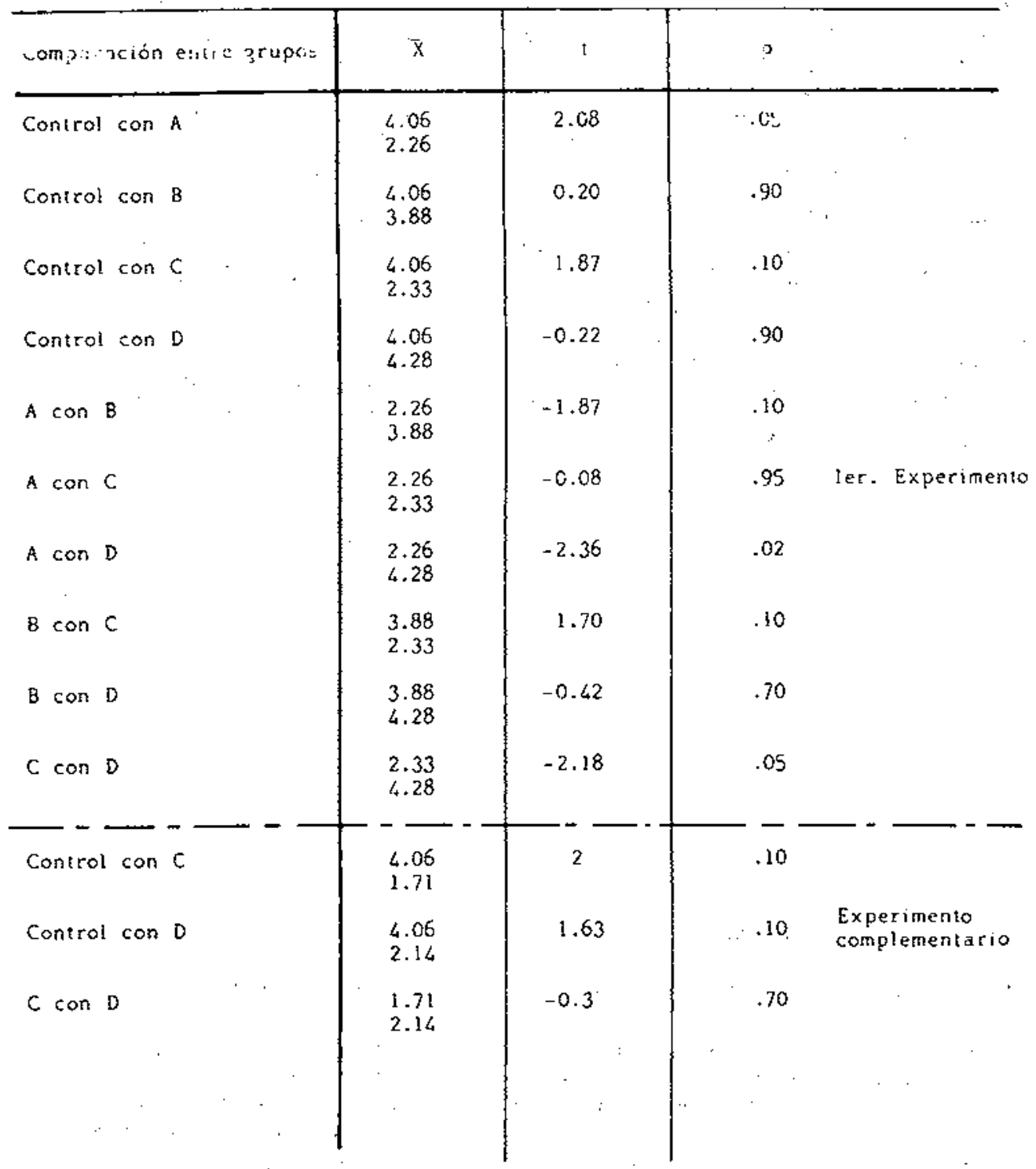

Fijándonos en la Tabla 1, vemos que los grupos que han producido los cambios más significativos son:

- Verbalización de la suma (A)

- Verbalización de la suma y del orden que debe seguirse para hacerla (C)

- El conjunto de (A) y (C), o sea, verbalización de la suma y del orden que debe seguirse; más la verbalización de la autocorrección (D).
Estos hallazgos nos hacen reparar en una constante que vemos en los tres procedimientos anteriores, que es: «la verbalización de la suma». Esta podría ser una variable importante ya que si analizamos los distintos procedimientos y sus efectos, observamos que en el grupo (A), que verbaliza la suma, obtenemos cambios significativos; en el grupo (B) que no verbaliza la suma sino el orden a seguir para hacerla, no obtenemos cambios significativos y, en cambio, el grupo (C) que tam- 
bién verbaliza la suma, encontramos nuevamente cambios relevantes con tendencia a la significación. Del grupo (D) podemos decir lo mismo que de los otros dos pero no podemos establecer la relación de forma tan clara, ya que el factor de verbalización de la autocorrección de las sumas, que forma parte de este procedimiento, creemos que también afecta a los resultados $o$, al menos, puede afectarlos.

La efectividad de la autoverbalización de las operaciones aritméticas ha estado informada también, como vimos, por Lovitt y Curtis (1968).

Por el contrario, encontramos resultados contradictorios, respecto a la "autoverbalización de la suma», informados por Spates y Kanfer (1977); y no observamos tampoco la superioridad del procedimiento de uestablecimiento de criterio", que es equivalente, en nuestro experimento, a la "verbalización del orden a seguir para hacer la suma» informado por los mismos autores.

Resumiendo el comentario hecho y, a modo de con-

\section{REFERENCLAS BIBLIOGRAFICAS}

BACKHOF, E., 1981, Gradación de sumas. (Entrevista personal).

BANDURA, A., y PERLOFF, B., 1967, Relative efficacy of self-monitored and externally imposed reiforcement systems. Journal of Personality and Social Psychology, No. $7, \mathrm{pp}, 111 \cdot 116$.

BANDURA, A., y KUPERS, C.J., 1963, Transmission of paterns of self-reiforcement through modeling. Journal of Abnormal and Social Psychology, No. 69, pp. 1.9.

BANDURA, A., 1969, Principles of behavior modification. (Nueva York: Holt, Rinehart and Winston).

BAYES R., 1980, Prólogo a la tercera edición. En Bayés, R. Una introducción al metodo cientlfico en psicologia. (Fontanella: Barcelona).

BIJOU, S.W., y BAER, D.M., 1961, Self-control. Child Development, Volum I: Asystematic and Empirical Theory. (Appleton-Century-Crofts; Nueva York). (Traducción Castellana: 1975, Psicologfa del desarrollo infantil. Teoría empírica y sistemática de la conducta. (Trillas: México).

BORNSTEIN, P.H. y QUEVILLON, R.P., 1976, The effects of a self-instruccional package on overactive pres- clusiones, podemos decir:

- Los procedimientos autoinstruccionales son efectivos para mejorar el aprendizaje de problemas aritméticos sencillos.

- La variable que se ha observado más relevante de los procedimientos autoinstruccionales es la «verbalización de la suma».

- Los resultados parciales obtenidos para Spates y Kanfer (1977) no coinciden con los de esta investigación.

- Enseñar a autoverbalizar las tareas a los escolares parece ser un buen método a anadir a la intervención educativa del maestro.

Finalmente, sólo nos resta decir que el estudio que hemos presentado quisiera contribuir, aunque modestamente, al cambio o mejor dicho a la evolución que ha iniciado el Análisis del Comportamiento en estos últimos años. chool boys. Journal of applied behavior analysis, No. 9, pp. 179-188.

BURGIO, L.D., WHITMAN, T.L., y JOHNSON, M.R., 1980. A self-instructional package for increasing attending behavior in educable mentally retarded children. Journal of applied behavior analysis. No. 13, pp. 443-459.

CAUTELA, J.R., 1966, Hipnosis and behavior therapy. Behavior, Research, and Therapy, No. 4, pp. 59-64.

CAUTELA, J,R., 1970, Covert reiforcement. Behavior Therapy. No. 1, pp. 33-50.

DENICOLA, J. y SANDLER, J., 1980, Training abusive parents in ment and self-control skills. Behavior Therapy, No. 11, pp. 263-270.

GENOVARD, C., GOTZENS, C. y MONTANE, I., 1981, Psicologla de la educacion (una perspectiva interdiscipli. nar). (Ediciones CEAC: Barcelona).

HOMME, L.E., 1965, Perspectives in psychology: XXIV. Control of coverants, the operants of the mind. Psychological Record, No. 15, pp. 501.511.

KANFER, F.H. y GOLDFOOT, D.A., 1966, Self-control and the tolerance of naxious stimulation. Psychological 
Reports. No. 18, pp. 79-85.

KANFER, F.H., 1970, Self-monitoring: methodological limitation and clinical applications. Journol of Consulting and Clinical applications. No. 35, pp. 148-152.

KANFER, F.H. y PHILLIPS, J.S., 1970, Learning fundations of behovior therapy. (John Wiley y Sons: Nueva York). (Traducción castellana: 1976. Principios de aprendizaje en la terapia del comportamiento. Trillas: México).

KANFER, F.H., 1975, Autocontrol en nithos: investigación y teorfa. En: V. Pelechano (ed.) ler. Simposium sobre aprendizaje y modificación de conducta en ambientes educativos. Servicio y publicaciones del ministerio de educación y ciencia).

KENDALL, P.C. y WICOX, L.E., 1980, Cognitivebehavioral treatment for impulsivity: concrete versus conceptual training in non-self-controlled problem children. Journal of consulting and clinical Psychology. No. 1, pp. B0-91.

LOVIT, T.C. y CURTIS, K.A., 1969, Academic response rate as a function of teacher and self-imposed contingencies. Journal of applied behavior analysis. No. 2, pp. 49-53.

LURIA, A.R., 1959, The directive funtion of speech in development. Word. No. 15, pp. 341-352.

LURLA, A.R., 1961, The role of speech in the regulation of normal and abnormal behavior (Liveright: Nueva York).

LURIA, A.R., 1975, Riech i myshlenie. (Ediciones de la Universidad de Moscú). (Traducción castellana: 1980, Lenguaje y pensamiento, Fontanella: Barcelona).

MAHONEY, M.J., 1970, Toward an experimental analysis of coverant control. Behavior Therapy. No. 1, pp. S10-521. (Traducción castellana: Hacia un analisis experimental del control de las operantes encubiertas. En: R. Ardila (ed.), 1980, Terapia del comportamiento: Fundamentos, técnicas y aplicaciones. Desclée de Brower: Bilbao).
MAHONEY, M.J., 1981, La relevancia de los procesos evolutivos para la psicoterapia. Análisis y modificación de conducta, No. 14 y 15 , pp. 155-170.

MEICHENBAUM, D.H. y GOODMAN, J., 1971, Training impulsive children to talk to themselves: a means of developing self-control. Journal of abnormal Psychology, No. 2, pp. 115-126.

MEICHENBAUM, D.H., 1981, Una perspectiva cognitivocomportamental del proceso de socialización. Análisis y modificación de conducta, Vol. 7, No. 14 y 15, pp. 85-113.

MISCHEL, W. y PATTERSON, CH. J., 1976, Sustantive and structural elements of effective plans for self-control. Journal of personality and social psychology, No. 5, pp. 942-950.

NEWMAN, A. y KANFER, F.H., 1976, Self-control in children: the effects of training under fixed, decreasing and increasing delay of reward. Journal of experimental child psychology, No. 21, pp. 12-24.

O'LEARY, S.G. y DUBEY, D.R., 1979, Applications of Selfcontrol procedures by children: A review. Journal of ap: plied behavior analysis, No. 12, pp. 449-465.

PINILlOS, J.L., 1975, Principios de psicología. (Alianza: Madrid).

RACHLIN, H., 1970, The self-control. Introduction to modern Behaviorism. (W. H. Freeman and company: San Francisco). (Traducción castellana: El autocontrol. Introducción al conductismo moderno, Debate: Madrid, 1977).

SANDERS, M.R. y GLYNN, T., 1981, Training parents in behavioral self-management: an analysis of generalitzation and maintenance. Journol of applaied behavior analysis, No. 14, pp. 223-237.

SPATES, CH. R. y KANFER, F.H., 1977, Self-monitoring, self-evaluation, and self-reiforcement in children's learnig: A test of a multistage self-regulation model. Behavior Therapy, No. 8. pp. 9-16. 